\title{
Editorial
}

\section{Using Brain Waves to Control Computers and Machines}

\author{
Christos Papadelis, ${ }^{1}$ Christoph Braun,, ${ }^{2,3}$ Dimitrios Pantazis, ${ }^{4}$ \\ Surjo R. Soekadar, ${ }^{5}$ and Panagiotis Bamidis ${ }^{6}$ \\ ${ }^{1}$ Harvard Medical School, Boston Children's Hospital, Boston, MA 02115, USA \\ ${ }^{2}$ MEG Center, Eberhard Karls University of Tübingen, 72076 Tübingen, Germany \\ ${ }^{3}$ Neuroimaging Laboratory, Center for Mind/Brain Sciences (CIMeC), University of Trento, \\ 38123 Mattarello, Italy \\ ${ }^{4}$ Magnetoencephalography (MEG) Laboratory, Martinos Imaging Center at Massachusetts Institute of Technology (MIT), \\ Cambridge, MA 02139, USA \\ ${ }^{5}$ Applied Neurotechnology Lab, Institute of Medical Psychology and Behavioral Neurobiology \\ and Department of Psychiatry and Psychotherapy, University of Tübingen, 72076 Tübingen, Germany \\ ${ }^{6}$ Laboratory of Medical Informatics, School of Medicine, Aristotle University of Thessaloniki, \\ 54123 Thessaloniki, Greece
}

Correspondence should be addressed to Christos Papadelis; christos.papadelis@childrens.harvard.edu

Received 26 May 2013; Accepted 26 May 2013

Copyright (C) 2013 Christos Papadelis et al. This is an open access article distributed under the Creative Commons Attribution License, which permits unrestricted use, distribution, and reproduction in any medium, provided the original work is properly cited.

Humans have traditionally interacted with computers or machines by using their hands to manipulate computer components. This kind of human-computer interaction (HCI), however, considerably limits the human's freedom to communicate with machines. Over the years, many attempts have been made to develop technologies that include other modalities used for communication, for example, speech or gestures, to make HCI more intuitive. Recent advances in cognitive neuroscience and neuroimaging technologies in particular have allowed for the establishment of direct communication between the human brain and machines. This ability is made possible through invasive and noninvasive sensors that can monitor physiological processes reflected in brain waves, which are translated online into control signals for external devices or machines.

Such brain-computer interfaces (BCIs) provide a direct communication method to convey brain messages to an external device independent from the brain's motor output. They are often directed at assisting, augmenting, or repairing human cognitive or sensory-motor functions. In BCIs, users explicitly manipulate their brain activity instead of using motor movements in order to produce brain waves that can be used to control computers or machines. The development of efficient BCIs and their implementation in hybrid systems that combine well-established methods in HCI and brain control will not only transform the way we perform everyday tasks, but also improve the quality of life for individuals with physical disabilities. This is particularly important for those who suffer from devastating neuromuscular injuries and neurodegenerative diseases which may lead to paralysis and the inability to communicate through speech or gesture.

In a BCI system, brain activity is usually recorded using a noninvasive neuroimaging technology, such as electroencephalography (EEG), magnetoencephalography (MEG), functional magnetic resonance imaging (fMRI), or nearinfrared spectroscopy (NIRS). In some cases, invasive technologies are used, such as electrocorticography (ECoG). In the majority of BCI systems, scalp EEG data are recorded, with the type of BCI system categorized based on the measure of brain activity used for BCI control. Each system has its own shortcomings and disadvantages. For instance, the information transfer rates of currently available noninvasive BCI systems are still very limited and do not allow for versatile control and interaction with assistive machines. 
Different BCI methods are sometimes combined in hybrid systems in order to improve accuracy, reduce errors, and overcome disadvantages specific to each conventional BCI system individually.

In this special issue, S. Amiri et al. review the literature on hybrid BCIs, pointing out their main advantages and disadvantages. By using brain waves in combination with other biosignals, performance in BCI control may be further improved, enhancing the ability of people with compromised motor systems to interact with the assistive machines. Next, P. R. de Almeida Ribeiro et al. provide an overview of the current state of assistive, noninvasive BCI research and propose to integrate brain waves and other biosignals, for example, electrooculography (EOG), for improved control and applicability of assistive machines in paralysis. In addition to introducing an example of such a system, they also discuss potential future developments.

P. Margaux et al. present an online P300-Speller employing automatic error correction. The system integrates error potentials that allow the recognition of misinterpreted commands in BCIs control. While at the group level, correction neither improved nor deteriorated spelling accuracy, automatic correction yielded a higher information transfer rate than a respelling strategy. Also, they found that the more the BCI user engaged in the task, the more useful and well accepted the automatic error correction was.

Studying motor imagery is important for developing high-performance BCIs. Motor imagery is the visualization and rehearsal of an imaginary movement as opposed to the actual execution of the movement. A. Athanasiou et al. studied event-related de/synchronization of the mu-rhythm (brain waves with a frequency of $8-15 \mathrm{~Hz}$ recorded over sensorimotor areas of the cortex) in the context of motor imagery performance. By using the eConnectome, they identified cortical activation in sensorimotor areas during actual and imaginary movements. In their study, R. Mohammadi et al. proposed pre- and postprocessing techniques to enhance the performance of BCIs for the onset detection of a mental task as a more general aspect of motor imagery.

Hardware components also play a crucial role in the development of BCIs. A. Astaras et al. developed and tested a prototype hybrid system consisting of two input devices and a robotic arm. The input devices were an exoskeletal positionsensing harness and a commercially available dry electrode BCI headset. All parts were designed using computeraided design (CAD) software, manufactured with computer numerical control (CNC) and laser cutting machines.

Beyond medical applications, BCI has also a great potential for gaming, a domain where users are open to novelty and eager to face new challenges. Implementation of gaming aspects into clinical BCI applications could prove useful by yielding better efficiency and wider acceptance of BCI-based therapies. E. Maby et al. present a BCI version of the famous game "Connect Four." Target selection was based on brain event-related responses measured with nine EEG sensors. Experimental evaluation on two competing healthy subjects yielded an average accuracy of $82 \%$ demonstrating that the BCI "Connect Four" can effectively be controlled.
These papers represent an exciting, insightful overview of the state of the art HCI technology, as well as emerging future topics in this important interdisciplinary field. We hope that this special issue will attract significant attention from peers working in all different fields related to HCI research, such as engineering, medicine, biology, psychology, and informatics, and that these reports will help chart new territory for the future of this growing field. 

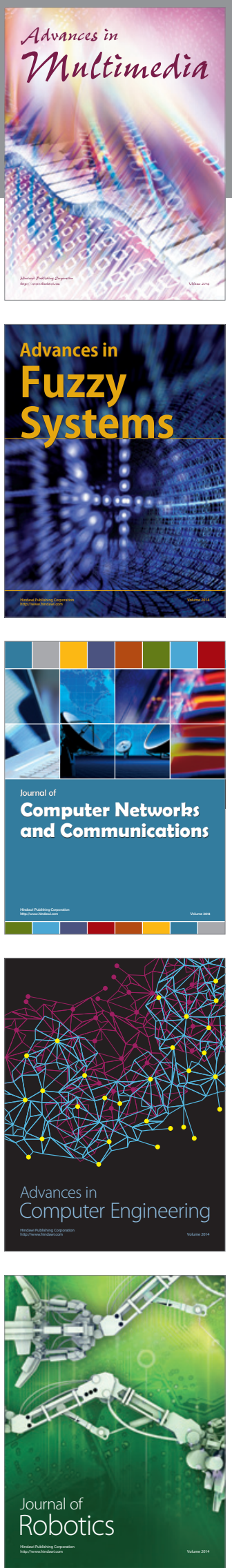

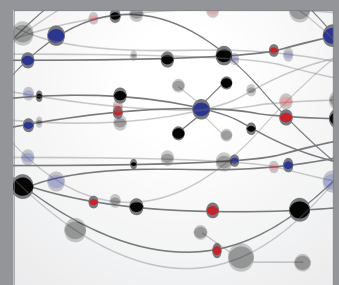

The Scientific World Journal
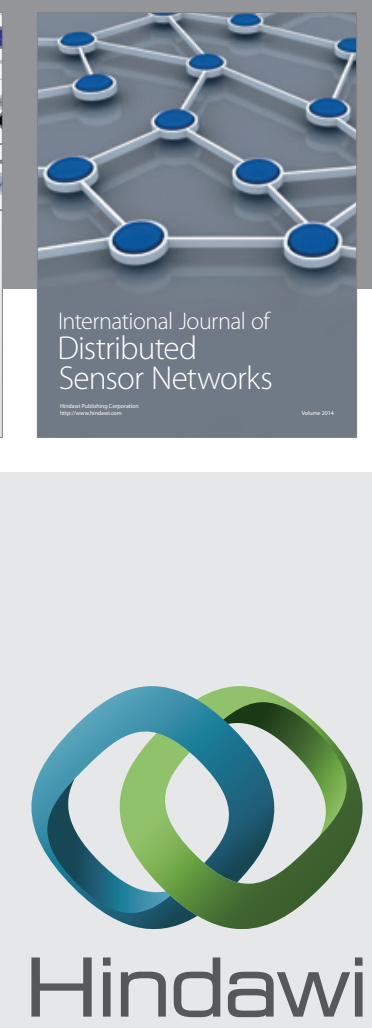

Submit your manuscripts at

http://www.hindawi.com
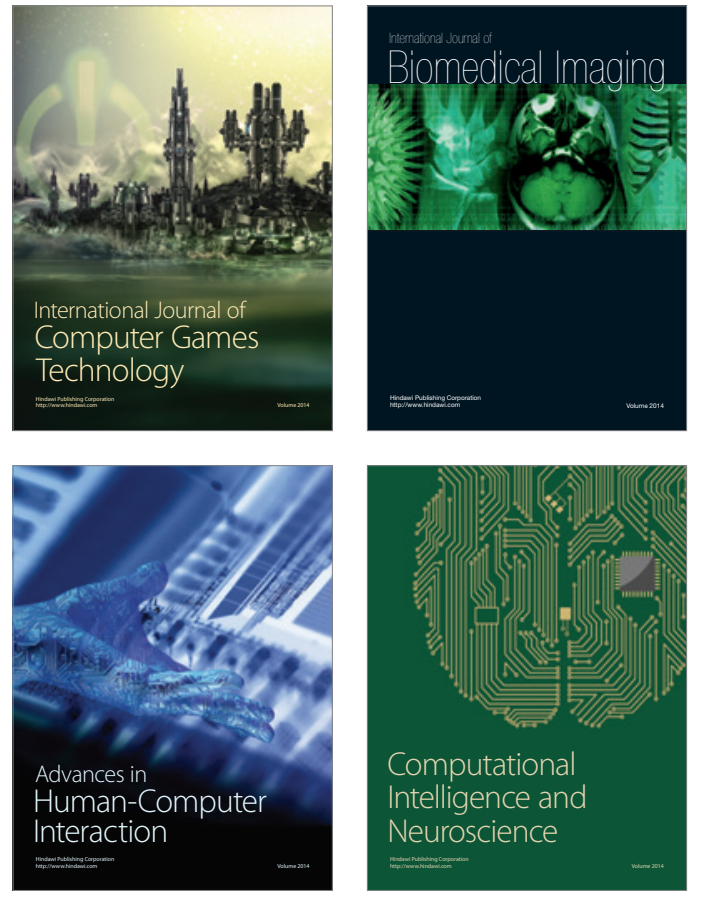
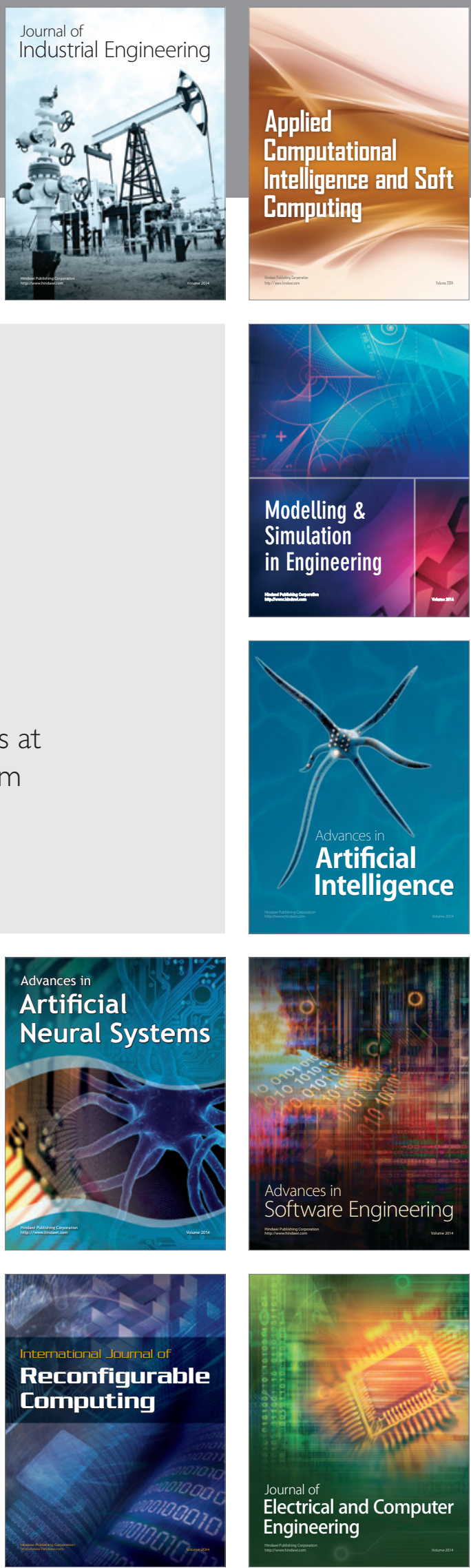